\title{
Survival of Infants Born at 23 Weeks of Gestation in a Tertiary Neonatal Unit
}

R Amoedo Blanco', S Zivanovic ${ }^{2}$, A Alarcon Allen ${ }^{2}$, M Anthony ${ }^{2}$, CC Roehr ${ }^{2}$

Sant Joan de Déu ${ }^{1}$ Neonatology department, Hospital Sant Joan de Déu , University of Barcelona, Spain

2 Neonatology department, John Radcliffe Hospital, University of Oxford, UK

\section{Introduction}

Prematurity is a main cause of mortality and morbidity in early childhood. Limits of viability in preterm infants are lowering continuously. As regards approach to the treatment of peri viable preterm infants in the UK, for infants born at 23 weeks gestational age (GA), parents are given the option of resuscitation and intensive care. However, the Nuffield Council on Bioethics Guidelines on Giving Intensive Care to Premature Babies (2006) do not mandate the treating physicians to provide or continue intensive care if they feel that this is not in the child's best interest.

Our aim was to analyze mortality before discharge and morbidities associated with death in preterm infants born at 23 weeks GA.

\section{Results}

\section{* A total of 54 infants were analyzed}

* Significantly more babies with a gestational age $<23+3$ weeks died $(16 / 34(47 \%)$ vs $5 / 20(25 \%)$, p 0.01)

* Persistent Pulmonary Hypertension in the Neonate (PPHN) was more frequent ( $31 \%$ vs $10 \%, \mathrm{p} 0.01$ ) in the group of infants who died

* There were no statistically significant differences in other infant characteristics (gender, mode of delivery, birth weight), perinatal management (antenatal steroids, support in delivery suit), treatment in NICU (ventilation, surfactant, inotropes, steroids, blood transfusions, antibiotics) and adverse events during hospital stay (pneumothorax, pulmonary haemorrhage, interstitial emphysema, atelectasis, PDA on ECHO, NEC Bell stage $>2$, IVH $>$ grade 2, jaundice, positive blood culture, late onset sepsis) between groups.

\section{Material and Methods}

* Retrospective study

* Infants of $23+0$ - 23+6 weeks GA, admitted to NICU at John Radcliffe Hospital, UK

* Study period June 2011 until February 2018

* Comparison: Infants who died before discharge and infants who survived and were discharged home

\section{Mortality of infants born at 23 weeks GA}

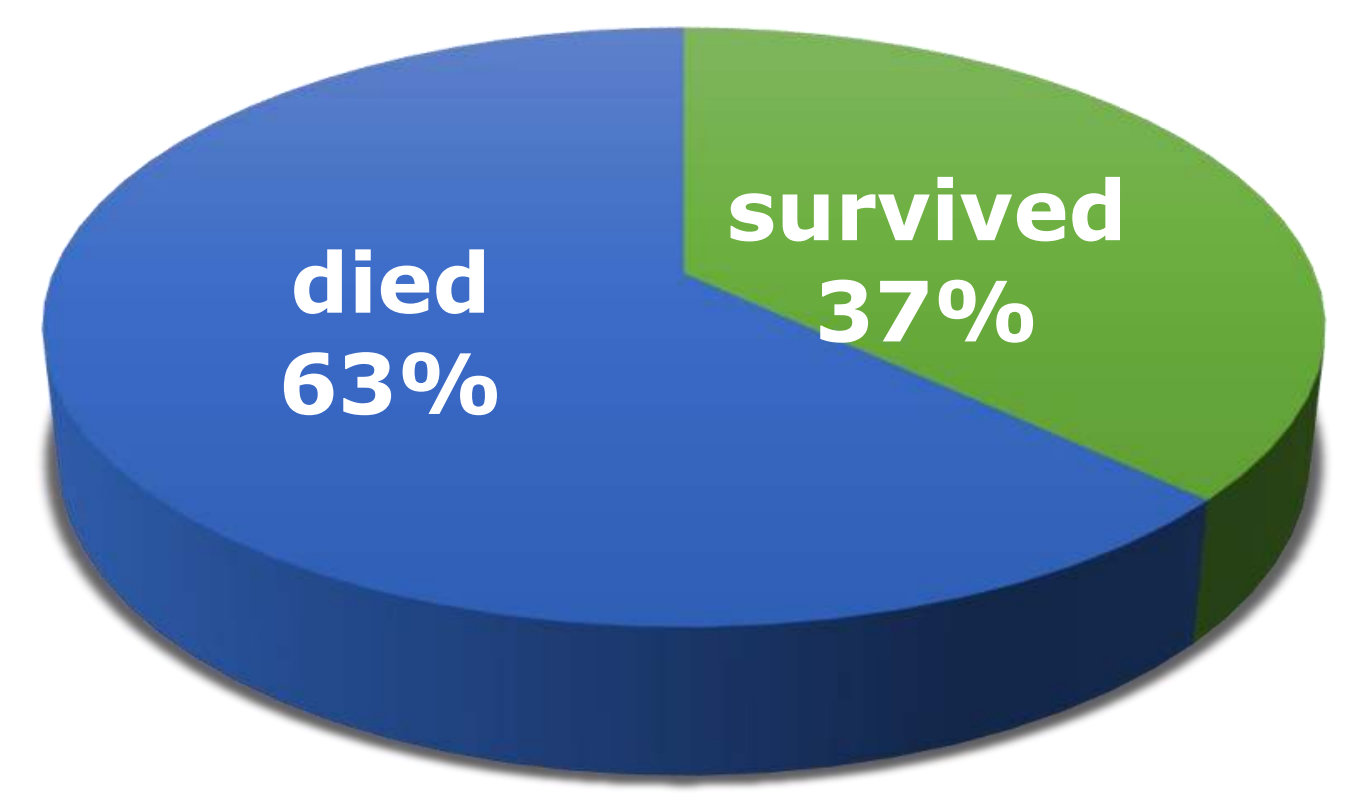

\section{Mortality of babies according to GA}

20

15

10

5

$\mathbf{0}$
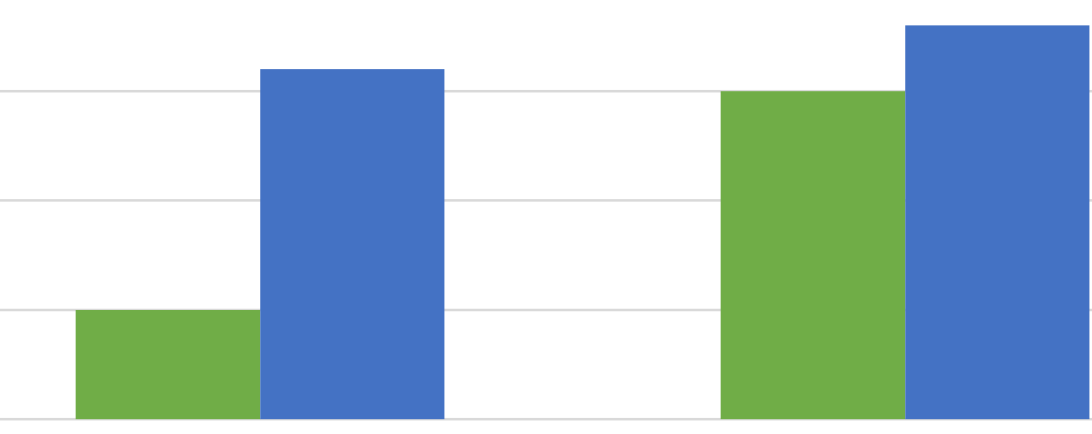

$23+0$ to $23+3 \quad 23+4$ to $23+6$

- Survived Died

\section{Conclusion}

In our cohort of infants, a GA lower than $23+3$ weeks was the main risk factor for death despite full provision of intensive care. PPHN was a significant, common complication in this group.

Analysis of outcomes of peri viable infants are important when considering local, national and international neonatal practice guidelines and when counseling parents. 